\title{
Health Monitoring Systems for Pregnant Women: Challenges and Issues
}

\author{
S. Veena ${ }^{1}$, D. John Aravindhar ${ }^{2}$ \\ ${ }^{I}$ Professor, CSE, S.A. Engineering College, Chennai, \\ ${ }^{2}$ Professor, CSE, Hindustan Institute of Technology and Science, Chennai
}

\begin{abstract}
It is important for all pregnant women throughout their gestational period to be able to access information, services and care by having direct communication with their doctors or their healthcare provider or their relatives. With the proliferation of mobile devices and the internet it has become very easy to get access to such information in a quick and efficient manner. There are also several awareness programs which teach about the right kind of physical activity and food to be followed by such pregnant women. Having access to such information helps these women identify and prevent any pregnancy related complications or risks which could lead to issues such as still-birth, pre term labor etc. Women who do not use this information wisely are likely to be putting their lives as well as the lives of their babies at risk. The main objective of this paper is to analyse various compound reasons pregnancy related issues and the technologies available to solve such issues.
\end{abstract}

Keywords: Bioinformatics, Data Analysis, Gestational period, Healthcare, Machine Learning, Pregnant women.

\section{Introduction}

With the spurt in the number of mobile devices, the internet and other communication technologies we now have a new sector which has emerged in the area of health care called as electronic health. We are looking at a sector which has a potential to deliver good quality healthcare at very low cost combined with a wonderful customer experience ${ }^{[1]}$ as well. It involves the exchange of medical information such as lab results, medical imaging, clinical information, prescriptions and dosages, past patient history to enable remote monitoring and on time determination of patient health status/critical diseases. With this technology we can potentially have access to automated and effective healthcare from anywhere ${ }^{[3]}$ on the globe with the least use of resources ${ }^{[2]}$ and power even in the most remote of locations where it is difficult to get in person medical consultation or support. With computers becoming smaller,networks becoming faster, mobile devices becoming smarter, reliable and secure, cost of making such devices decreasing, we are now provided with a variety of options to monitor our health remotely and effectively. But all this technology does not help unless we have a proper network of the healthcare professionals such as doctors, hospitals, nurses etc who can be connected with the patients thus enabling effective interaction between them and also increase the awareness of health issues/risks which was not possible before. There can be many types of networks that we build in our lives which help in this case. Some of the different networks that include the professional network of colleagues and customers, social networks which includes our friends, acquaintances and family networks that include our close relatives and family members. The electronic health sector enables connecting these networks to the network of healthcare professionals to enable faster, effective health care which can improve the areas of personal health, child health, geriatric health etc. These networks can increase the awareness of patients on the potential health issues/risks and remediation. This was not previously possible unless there was a face to face interaction between the patient and healthcare professionals ${ }^{[5]}$.

Today a very small percentage of pregnant mothers know about the development status of their baby ${ }^{[6]}$. They are not aware of the stages of pregnancy and the parameters that need to be measured or tracked during these various stages. They are also not aware of what are the right foods and the wrong foods to eat during their 
pregnancy. The electronic health sector will allow these mothers to have access to such data about the various stages of pregnancy and also allow them to connect to this network of healthcare professionals to get their doubts clarified and any emergencies taken care of. This capability is enabled by the use of Smartphone and internet which enables this information to be available at the right place at the right time at the right stage of development. Having such a connected network also enables the Hospitals/nursing homes to monitor and attend to any urgent needs of such pregnant women which can reduce the delay in taking certain decisions which can otherwise endanger the lift of the mother and the baby as well. This will improve the mother mortality rate and also improve the fetal mortality rate while improving the general health/survival of the women.

Literature Review: Vyacheslav Shulgin ${ }^{[7]}$ The problem of premature or preterm birth is a major cause of baby's long term morbidity and also a primary cause of neonatal fatalities as well. The current devices and method used for monitoring and predicting preterm births are very inaccurate, invasive, expensive and can only be used in hospitals/clinical laboratories. One of the newer method which have been discovered to improve on this is to analyze the Electrohysterography signals (EHG) which is measured from the abdominal region of pregnant women. These signals are said to be very effective in predicting preterm births. The most important part of the signals collected is the Conduction Velocity (CV) which occurs during uterine contraction. The paper discusses an algorithm which can be used to estimate the CV based on the space-time processing of abdominal signals. This algorithm has been formulated on the basis of actual EHG recordings to estimate the $\mathrm{CV}$.

YunusSantur $^{[8]}$, People today use the internet for almost everything in their life such as collecting information, socializing with people, being in touch with family and friends and to handle any official business as well. In addition to connecting offline to such professional, personal and social networks a lot more people are now connected to virtual social networks. Facebook is the world's largest social network and has so many people who are on their network and who have formed virtual networks with other people/companies on the same network. There are millions of such social networks available on various topics which can be professional, personal, social. Imagine if we could set up such a social network which would connect pregnant women with other pregnant women or women who have been through pregnancy. This network can be used to share useful information about the various stages of a babies development, what to eat and not to eat, what are the risks to be monitored, where to get the right information/help, when to take regular scans, when to give vaccinations to the baby, what is the weight/height at various stages of the baby etc. This would really help spread the awareness of pregnancy related risk and the steps to be taken to ensure the wellbeing of both the mother and the baby.

Lingyun $\mathrm{Wu}^{[9]}$ Ultrasound (US) images are very important to monitor the growth of the baby during the gestational period of a pregnant woman. It helps determine the size/weight/heart rateetc of the baby based on which we can determine whether the baby is healthy or not. It can also help identify abnormalities such as missing organs, double organs, deformities etc very early so that the necessary decisions can be taken on whether to abort the baby or to continue with the pregnancy. In order to make such critical decisions the quality of the ultrasound images must be very good. But the process of getting good quality images is very manual and labor intensive and all of this can only happen in a hospital/ clinical environment. In order to improve the image quality, reduce the measurement error, improve the slice choice and the accuracy of measurement, a new method was proposed to assess the ultrasound Image quality. This is accomplished by using the concept of Artificial Neural Networks. There are 2 custom deep learning models that were developed denoted as L-CNN and C-CNN. The former aims to seek out the ROI (Region Of Interest) which is then used by the latter to evaluate the quality of the images and assess the details of the required parts of the body that need to be measured. In order to improve the performance of the models, we need to complement the neural network with local features derived from the original data. More the number of input sources the better is the quality of the image. This method has also been evaluated by a group of medical doctors and has found to be on par with the results from actual doctors.

Nandakishor D Valakunde ${ }^{[10]}$, India has more number of stillbirths and a higher maternal mortality rate compared to other developing countries of the world. It has been determined that a pregnant woman should make at least four visits to their doctor or their healthcare provider in order to ensure a safe pregnancy for both the mother and the baby. The Smart ASHA Pregnancy Monitoring System [SAPMS] is one such system which 
was created to reduce the incidents of maternal mortality by enabling healthcare providers to reach out to the pregnant women in the most remote/underprivileged parts of the world which is made possible by the many types of mobile devices (smartphones) and the internet. This system can provide efficient and effective healthcare to the pregnant women.

Raghav Hari Krishna V S ${ }^{[11]}$ The most prevalent reason for perinatal morbidity is preterm birth. In order to be able to correctly predict preterm we need to collect a lot of information such as the mother's height, weight, gravida or number of pregnancies and para. There is a model which has been developed which can predict the risk of pre term. The authors were able to achieve $89.99 \%$ accurate results which is very promising. The model developed can then be used to predict/identify the women.

\section{Challenges:}

- To facilitate a communication network between the healthcare providers and the patients.

- To facilitate in forming a communication network between the pregnant ladies and allows them to exchange their experiences and ideas.

- To facilitate in increasing the awareness around healthy food/healthy habits to pregnant women.

- To facilitate monitoring using the pregnant woman's health status using a wearable smart watch sensor connected to a mobile application.

- To facilitate monitoring the fetal movements using a sensor belt which can count the number of kicks.

- To facilitate delivering medical services to clinics in remote areas which are not accessible for in person consultation/support.

\section{Health Care Monitoring Systems For Pregnant Women:}

Health Care provider unit: This unit consists of a mobile application and also a web application using which doctors/nurses/healthcare providers can interact with their patients and diagnose any issues remotely. The healthcare providers can also use this network for creating awareness and to schedule any follow ups required. This unit creates a private social network which can be used to only connect the doctor/healthcare provider directly to the pregnant woman. This network can also be used to connect this unit to other pregnant women so that they can interact with and understand from each other. Doctors/healthcare providers can also post messages, images, videos etc which can then be used to create awareness with pregnant women using mobile application/SMS. In order to prevent incorrect diagnosis or unwanted images from being stored, there should exist a capability to be able to delete any incorrect images/files etc. This system will also provide full access to the pregnant woman's profile, history of treatments etc to enable giving the right support. The unit can also measure information about the physical activity of the mother and child using a wrist bond and also include the physical activity of the baby by measuring the number of kicks of the baby using a body touch sensor. ${ }^{[12]}$

Wearable Technology and Smartphones to Monitor Hypertension during Pregnancy: The authors talk about being able to use wearable devices ${ }^{[19]}$ such as smart watches, wrist bands etc to measure, monitor and control hypertension during pregnancy which often leads to complications such as preeclampsia which can cause complications such as pre-tirm, still birth, miscarriage etc. By using this technology we can ensure that patients are engaging in healthy activities in turn reducing the costs associated with fetal and maternal health problems.

In addition to using wearables, we can also use smartphones ${ }^{[20]}$ to do the similar kind of monitoring. Today smartphones have become very versatile and have several sensors such as heart rate sensor, blood pressure sensor, fingerprint sensor etc which can be used to measure the required parameters of the pregnant woman. The data collected from the smartphones can also be used to perform analysis of the health status and share the same with the healthcare provider remotely.

\section{Measuring Uterine Contractions using On Body} Sensors: This paper proposes a cost effective system to monitor the risks of premature labor in pregnant women $^{[21]}$. As per their research premature birth is the most important reason for deaths of children before the age of 5 or even if they survive they will have issues such as hearing disability or loss of vision or mental instability. Their system works by measuring uterine contractions using a non-invasive body sensor which is then connected to a smartphone which will send an intimation/alert to a doctor/family member in case of emergency so that they can take care of the same.

Fetal Remote Monitoring System: This paper proposes a method to remotely monitor the status of 
pregnant women using several sensors which can be used to measure the below parameters.

- Heart Rate

- Abdominal Breathing

- Blood Pressure

- Fetal Movement Data

- Mother Movement Data

- Temperate of Mother

The parameters above can be measured by using non-invasive body based sensors ${ }^{[22]}$. The data from these sensors are collected, analysed and compared with previously collected data to determine the status of the mother/fetus. Based on the status the doctor/healthcare professional can take the required action.

Cloud Based HealthCare System: This paper proposes a cloud based infrastructure to collect and store electronic health records of patients which can then be used to perform analysis using machine learning to determine the status of the patient being monitored ${ }^{[23]}$. The data in the cloud can be collected either in real time from sensors worn by the patient or can also be collected from the health records of the patient shared by the hospital or by data collected in the past for the same patient. The information collected from the sensors are raw data which must then be processed to derive the right kind of information.

Intelligent Assistant for Pregnancy: This system enables remote monitoring ${ }^{[12]}$ of pregnancy related risks which is essential for women who are unable to get access to proper healthcare during their pregnancy which can result in complications such as preterm/stillbirth. The intent of this system is to monitor the health status of the pregnant woman using a network of on body non-invasive sensors connected to the internet. The data collected from these sensors is placed into a central repository. This information can then be compared to the previous information collected for the same patient and also information collected from other patients to determine whether the health status of the pregnant woman is fine or not. By using the internet this system helps connect the pregnant women to their doctor/ healthcare provider or family members to provide assistance during various stages of pregnancy. This network enables us to get real time suggestions from the doctor/healthcare provider. This system incorporates a lot of personalized information which is useful for the pregnant women such as the stages of delivery, due date, personalized diet recommendations etc. it can also track the weekly progress and any abnormalities in the pregnancy.

The system works by having doctors/hospitals/ patients register themselves to a network. The pregnant women have sensors/smartphones to measure critical parameters such as temperate, heartrate, blood pressure etc. The information collected is then stored in a cloud over the network. This information can then be analyzed using machine learning algorithms to determine the health status of the pregnant woman. This information can then be shared with the doctor/healthcare professionals or family members to identify any complications or risks based on which some urgent action needs to be taken. The information collected during registration of the patient would include the previous health details, previous scan results, any medications currently being consumed, any known family history, any known allergies etc. This information is very important to determine the health status and also for a doctor to understand the reasons behind a particular health status and to prescribe the right medicine for the same. This system will also be able to communicate the information collected regularly over the gestational period to their doctor, healthcare provider or family member either through mobile app based notifications or using SMS (Short Messaging Service). It can also send reminders of health checks/ scans etc. All of this will enable a pregnant woman to have access to the right information at the right time and thus prevent any pregnancy related risks that would normally have occurred due to the lack of awareness of the same.

\section{Conclusion}

It is important for all pregnant women throughout their gestational period to be able to access information, services and care by having direct communication with their doctors or their healthcare provider or their relatives. With the proliferation of mobile devices and the internet it has become very easy to get access to such information in a quick and efficient manner. This paper provides a summary of all such systems which exist to help provide such information to a pregnant woman in the most simple, cost effective manner.

\section{Conflict of Interest: Nil}




\section{Ethical Clearance:}

- This manuscript in part or in full has not been submitted or published anywhere.

- This manuscript does not contain previously published content.

\section{Source of Funding: Self}

\section{References}

1. Huberty J, Rowedder L, Hekler E, Adams M, Hanigan E, McClain D. "Development and design of an intervention to improve physical activity in pregnant women using Text4baby", Transl Behav Med. June 2016;6(2):285-94.

2. Doran F, Davis K.,"Factors that influence physical activity for pregnant and postpartum women and implications for primary care”.Aust J Prim Health. 2011;17(1):79-85.

3. Leite CF, do Nascimento SL, Helmo FR, Dos Reis Monteiro MLG, Dos Reis MA, Corrêa RRM, "An overview of maternal and fetal short and long-term impact of physical activity during pregnancy", Arch Gynecol Obstet. February 2017;295(2):27383.

4. R. Sameni, C. Jutten, and M. B. Shamsollahi, "Multichannel electrocardiogram decomposition using periodic component analysis." IEEE Trans. Biomed. Eng.,August 2008; 55(8): 1935-1940.

5. Sameni R, Jutten C, Shamsollahi M. B. "A deflation procedure for subspace decomposition", Signal Processing, IEEE Transactions. 2010; 58: 23632374.

6. Lakhno, "Autonomic imbalance captures maternal and fetal circulatory response to pre-eclampsia", Clin. Hypertens., 08 February 2017, 23: 5.

7. Vyacheslav Shulgin, "Spatio-Temporal Signal Processing for Fetus and Mother State Monitoring During Pregnancy", IEEE. May 2018;24-27..

8. YunusSantur, "Smart Pregnancy Tracker System Using Social Knowledge Networks for Women", IEEE.2017.

9. Lingyun $\mathrm{Wu}$, "FUIQA: Fetal Ultrasound Image Quality Assessment With Deep Convolutional Networks", IEEE. May 2017

10. Nandakishor D Valakunde, "Smart ASHA Pregnancy Monitoring System”, 2017 International Conference on Big Data, IoT and Data Science
Medico-legal Update, April-June 2021, Vol. 21, No. 2 1047 (BID) Vishwakarma Institute of Technology, Pune, Dec 20-22, 2017.

11. Raghav Hari Krishna V S, "Prediction of Preterm Pregnancies using Soft Computing Techniques Neural Networks and Gradient Descent Optimizer", (ICCCI -2018). Jan. 04 - 06, 2018.

12. Sabiha Khanum, "Designing a Pregnancy Care Network for Pregnant Women”.MDPI 2017.

13. Lao TT, Ho LF. "Relationship between preterm delivery and maternal height in teenage pregnancies", Department of Obstetrics and Gynaecology, The University of Hong Kong, People's Republic of China. 2000 Feb; 15(2): 4638.

14. U. Elaveyini. S. Prasanna Devi. K. Suryaprakasa Rao. "Neural Networks prediction of preterm delivery with first trimester bleeding", Archives of Gynecology and Obstetrics. 7 May 2010. SpringerVerlag 2010

15. J. Sikora, A. Matonia, R. Chabanskiy. "Recognition of Premature threatening labour symptoms from bioelectrical uterine Activity signals," Archives of Perinatal Medicine. 2011: 97- 103

16. Etemadi, M., Chung, P., Heller, J. A., Liu, J. A., Rand, L., and Roy, S. "Towards Birth Alert-a clinical device intended for early preterm birth detection", IEEE Trans Biomed Eng. July 2013;60(12): 3484.3493.

17. Conde-Agudelo, A. and Romero, R. ."Prediction of preterm birth in twin gestations using biophysical and biochemical tests", Am J Obstet Gynecol. Dec 2104; 211(6):583.595.

18. S. G. Santur, Y. Santur, M. Karaköse. "Knowledge Mining Approach For Healthy Monitoring From Pregnancy Data With Big Volumes", IJISAE, 2016; special issue-1:145-148.

19. Betsy DiamarBalbin Lopez, Diego Antonio Reyes Coronado, Jimmy Alexander Armas Aguirre and Paola A. Gonzalez. "Wearable Technology Model to Control and Monitor Hypertension during Pregnancy".

20. George K. Endo, Ibukun Oluwayomi, Victor Alexandru, YashodhanAthavale, Sridhar Krishnan. "Technology for Continuous Long-term Monitoring of Pregnant Women for Safe Childbirth".

21. Hisham Allahem and Srinivas Sampalli. "Framework to Monitor Pregnant Women with 
a High Risk of Premature Labour Using Sensor Networks".

22. Yu. A. Zhivolupova, Yu. O. Bobrova. "The Concept of the Fetal Remote Monitoring System Development".
23. Sepideh Poorejbari, Hamed Vahdat-Nejad. "An Introduction to Cloud-Based Pervasive Healthcare Systems". 